\title{
Radiologists virtually unwrap mummy's secrets
}

I n 1997, a radiologist in Montréal, Quebec examined a woman and found that her left femur had once been broken. That finding is now under suspicion. The radiologist said the woman's bad teeth stemmed from too much beer. That also appears to be wrong. A recent computed tomography (CT) scan has revealed much about the health of this mysterious woman and she only waited 2500 years for the exam. Give or take a century.

On the evening of Nov. 18, 2008, radiologists at the University of Ottawa Heart Institute slid Hetep-Bastet into a scanner's narrow core. Accustomed to tombs, Hetep-Bastet, an Egyptian mummy dating to sometime before 500 BC, didn't seem to mind. Dr. Andrew Nelson, the University of Western Ontario anthropologist interpreting the re- sults, says the radiological study of mummies teaches us about the history of diseases and the general health of past cultures.

Not that academic casket-cracking is new. Researchers have been gleaning health information from linen-wrapped remains for more than a century. The Museum of Natural History, in Austria, X-rayed an Egyptian coffin (W. König 14 Photographien mit RöntgenStrahlen, aufgenommen im Physikalischen Verein, Frankfurt a M. Leipzig: JA Barth, 1896) only a year after physicist Wilhelm Röntgen had taken the world's first x-ray. Many researchers have since studied mummies, often through autopsy. That was a problem, however, because autopsies made a mess of millennium-old remains.

"The real emphasis now is to do things non-destructively," says Nelson. "The CT scanner is ideal for that."

Technology no doubt makes investigations safer for King Tut and cohorts, but mummy-scanning offers new challenges for researchers. A CT scan of a living person is easy to interpret because bone is much denser than live tissue. Bones hungrily absorb radiation. Muscles, fat and organs are x-ray thoroughfares. But the thoroughly dehydrated skin and flesh of a mummy is similar in density to bone. Even the wrappings eventually come to resemble fibulas more than fabric. It is also impossible to inject dye into a mummy's bloodstream to highlight blood vessels, because mummies have no blood to stream.

Conducting the scan is a breeze. Technologists scanning a living patient must compensate for movement due to

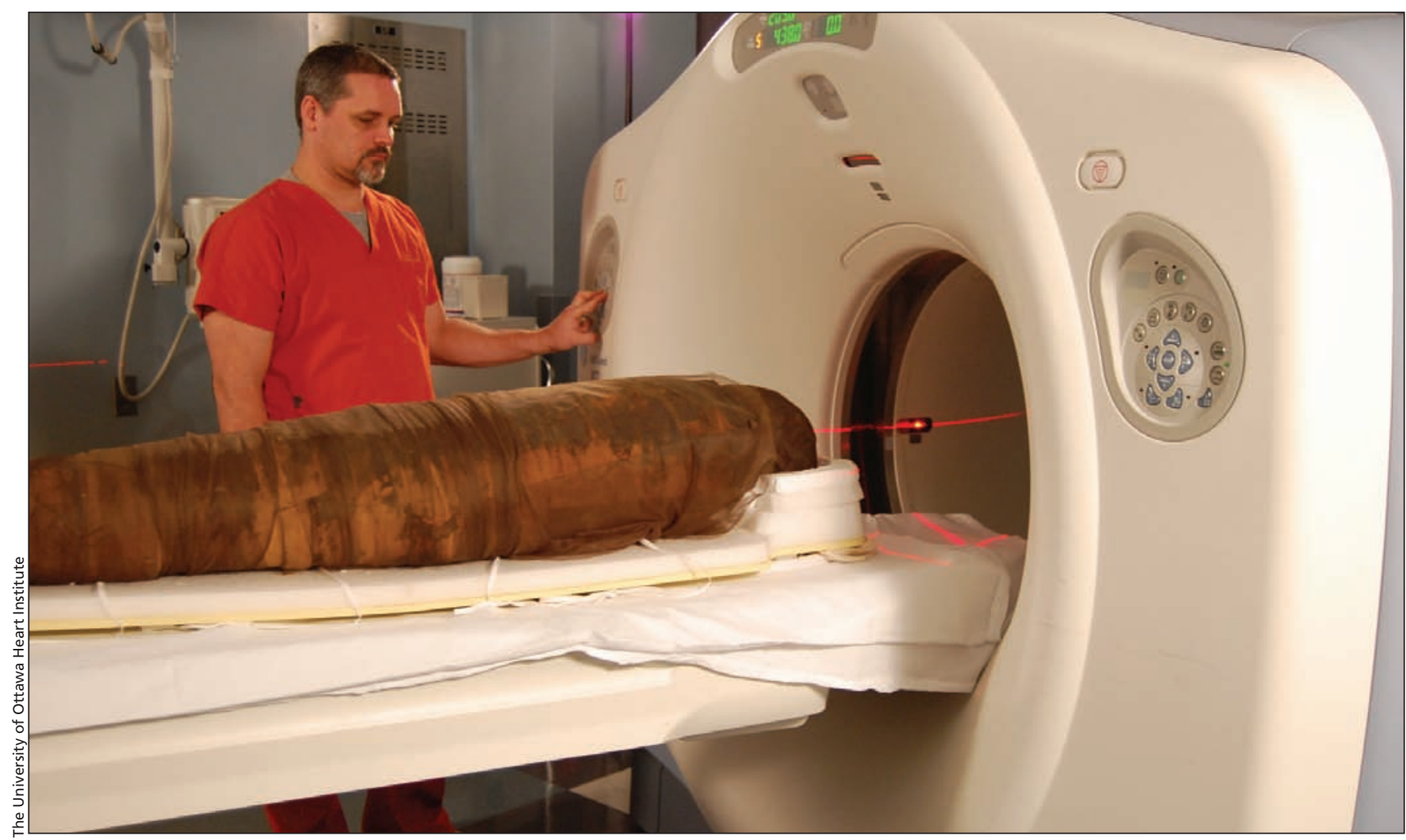

A computed tomography scan of Hetep-Bastet, an Egyptian mummy, was performed at The University of Ottawa Heart Institute on Nov. 18, 2008. Radiologists donated their time, and the scan took place after regular hours as to not disturb patient scans. Scanning is a nondestructive way to study ancient remains. 
breathing, fidgeting and a beating heart. But mummies tend to remain still. They also don't mind being bathed in radiation, so they remain in the scanner longer, allowing for better images. Capturing the data is easy. Creating an image from that data? Not so easy.

"There's only a few seconds to several minutes of reconstruction for a living patient," says Dr. Blair Macdonald, a staff radiologist with The Ottawa Hospital. "For a mummy, it could be in the range of hours to days to strip away various resins and identify what is soft tissue and bone."

Fortunately, a new way of conducting CT scans is simplifying that process. The dual-energy technique, used in the Hetep-Bastet exam, involves scanning at different power levels. This allows radiologists to capture more detailed information and better discriminate between bone and soft tissue. Since all parts of mummy remains are alike in density, they are ideal for testing the technique. Radiologists can then apply the lessons learned to living patients.

"The thing that is most exciting for me is the dual-energy scanning," says Macdonald, who assisted in the HetepBastet scan. "We are only just breaking the surface of that."

Nelson's preliminary report on Hetep-Bastet - who is on display at the Canadian Museum of Civilization in Gatineau, Quebec, until Aug. 16 both confirms and challenges findings of an old x-ray exam. Nelson agrees with the 1997 findings by a Montréal radiologist that Hetep-Bastet was a woman with poor dental health. But he could not verify that she had a healed leg fracture. Nor did he find evidence

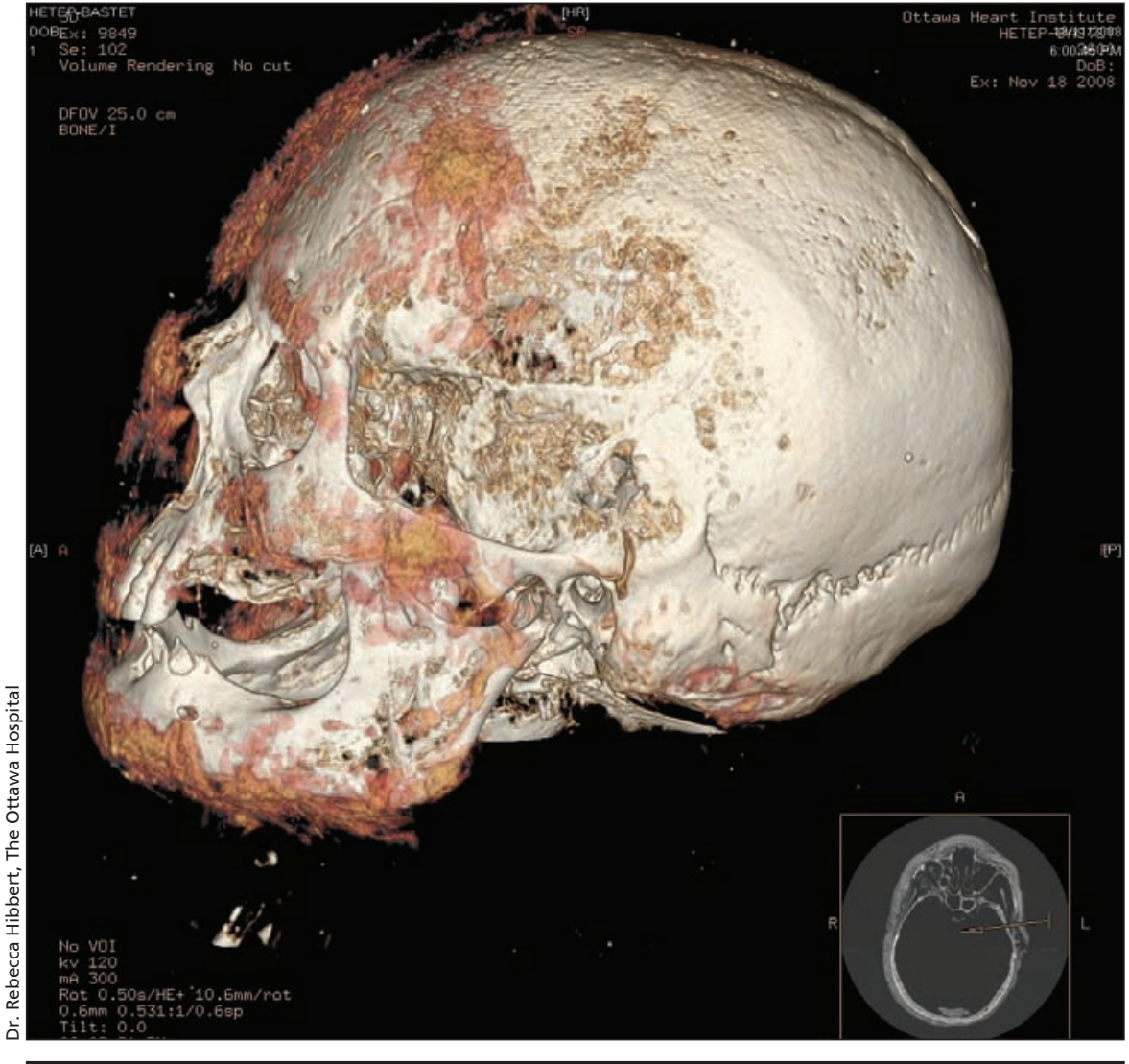

The scan indicated that Hetep-Bastet, a woman dating to sometime before $500 \mathrm{BC}$, had poor dental health, perhaps because of a high-carb diet and wear from too much sand in her bread dough.

that Hetep-Bastet's dental woes resulted from beer. The more likely cause, says Nelson, was a high-carb diet — and too much sand in the bread dough.

It is difficult, however, to conclude much about a society by examining just one of its members. Nelson advocates for a worldwide database of mummy scans, and has applied for a Canada Foundation for Innovation grant to set one up. Finding money to pay for the scans will likely be more challenging than finding help to conduct them. People in many fields - from radiology to archaeology to computer science jump at the chance to scan a mummy, says Nelson.

'It's just a matter of working out the dates and the mechanics of getting it to the hospital." - Roger Collier, CMAJ

DOI:10.1503/cmaj.090237 